\title{
Noise Whitening in Airborne Wind Profiling with a Pulsed 2-Micron Coherent Doppler Lidar at NASA Langley Research Center
}

\author{
Jeffrey Y. Beyon ${ }^{1 *}$, Grant E. Arthur ${ }^{2}$, Grady J. Koch ${ }^{3}$, and Michael J. Kavaya ${ }^{4}$ \\ ${ }^{1}$ NASA Langley Research Center, MS 488, Hampton, VA 23681, USA, Jeffrey.Y.Beyon@nasa.gov \\ ${ }^{2}$ Electrical Engineering Department, Harding University, Searcy, AR 72149, USA, grantarthur5@gmail.com \\ ${ }^{3}$ NASA Langley Research Center, MS 468, Hampton, VA 23681, USA, Grady.J.Koch@nasa.gov \\ ${ }^{4}$ NASA Langley Research Center, MS 468, Hampton, VA 23681, USA, Michael.J.Kavaya@nasa.gov
}

\begin{abstract}
Two different noise whitening methods in airborne wind profiling with a pulsed 2-micron coherent Doppler lidar system at NASA Langley Research Center in Virginia are presented. In order to provide accurate wind parameter estimates from the airborne lidar data acquired during the NASA Genesis and Rapid Intensification Processes (GRIP) campaign in 2010, the adverse effects of background instrument noise must be compensated properly in the early stage of data processing. The results of the two methods are presented using selected GRIP data and compared with the dropsonde data for verification purposes.
\end{abstract}

Keywords: Noise whitening, coherent lidar, GRIP, wind profile, DAWN AIR

\section{INTRODUCTION}

The fundamental concept of wind profiling based on lidar returns is Doppler shift (DS) estimation in wind speed and direction estimation using more than one line of sight (LOS) of lidar beam [7]. In DS estimation, it involves the spectral analyses of lidar returns using fast Fourier transform (FFT). The DS estimation is closely related to the peak detection process of lidar return spectra, which are corrupted by various kinds of noise, most eminently the instrument noise. This paper discusses the adverse impacts of the background noise in peak detection, remedies to compensate them, and samples of the results of such compensations. As part of such efforts, two noise whitening methods are presented and their impacts are shown in final wind products. The lidar data were acquired by the Doppler Aerosol Wind (DAWN) AIR lidar instrument during the summer of 2010 as part of the NASA Genesis and Rapid Intensification Process (GRIP) hurricane campaign. The general overview of the data acquisition and processing software is also presented in this paper [1-2].

\section{AIRBORNE WIND PROFILING SOFTWARE FOR DAWN AIR}

The software that controls the data acquisition, processing, and display system (DAPDS) is called data acquisition and processing software (DAPS), and was written in ANSI-C using National Instruments' LabWindows/CVI compiler. Its operation time during the GRIP campaign was approximately 130 hours. The mission was to measure the wind using the Langley's DAWN AIR lidar instrument while aboard NASA’s DC-8 flying laboratory.

The GRIP hurricane campaign involved three aircraft platforms: DC-8, WB-57, and Global Hawk Unmanned Airborne System (UAS). The DC-8 was based in Ft. Lauderdale, FL, WB-57 in Houston, TX, and the Global Hawk flew out of NASA Dryden Flight Research Facility in CA. The deployment period was August 15 through September 20, 2010. The GRIP campaign focused on the upper level hurricane measurements while flying through and above hurricanes during the campaign period.

*Jeffrey.Y.Beyon@nasa.gov; phone 1757 864-4249; fax 1757 864-7944; www.nasa.gov 
DAPS is in charge of controlling the scanner, digitizer, and the execution of real-time wind profiling algorithms while acquiring lidar returns at $500 \mathrm{MHz}$ sampling rate. The trigger rate was $10 \mathrm{~Hz}$ at which 55,000 samples (or more) were

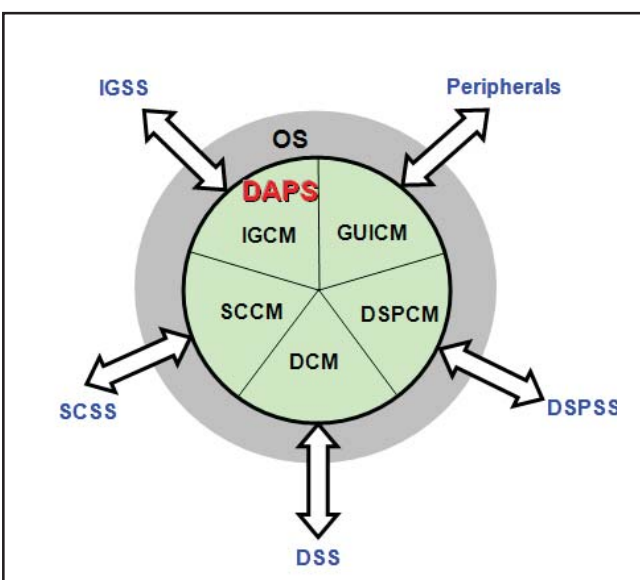

Fig. 1. Anatomy of DAPS acquired for each trigger pulse. Figure 1 shows the anatomy of DAPS where the five control modules constitute the core functionalities of DAPS. They are INS/GPS control module (IGCM), graphic user interface control module (GUICM), digital signal processor control module (DSPCM), digitizer control module (DCM), and scanner control module (SCCM) and the arrows indicate the subsystems (SS) that each control module is associated with. DAPS runs in the environment of Windows XP on a 6U compact PCI chassis platform.

A brief description of a typical operation scenario of DAPS is as follows assuming the lidar system is airborne and ready to lase: It checks if the digitizer is calibrated and ready for digitization. If not, it executes a calibration routine, then arms the digitizer waiting for a series of external TTL pulses. DAPS also allocates and optimizes the CPU memory and the DSP memory for lidar time series data. When an external TTL pulse arrives, DAPS commands the digitizer to initiate the data acquisition process on the rising edge of the TTL pulse, which runs at $10 \mathrm{~Hz}$. Before the next TTL pulse arrives, DAPS performs spectral analyses utilizing the DSP subsystem (DSPSS). When this process repeats for a user selected nominal times, DAPS commands the scanner control subsystem (SCSS) to move the telescope to the next look direction and repeats the same process. The number of look directions during the GRIP campaign was five (two larboard/port, straight ahead, two starboard). When one pattern of scanning all five LOSs is complete, the raw and processed lidar data are archived.

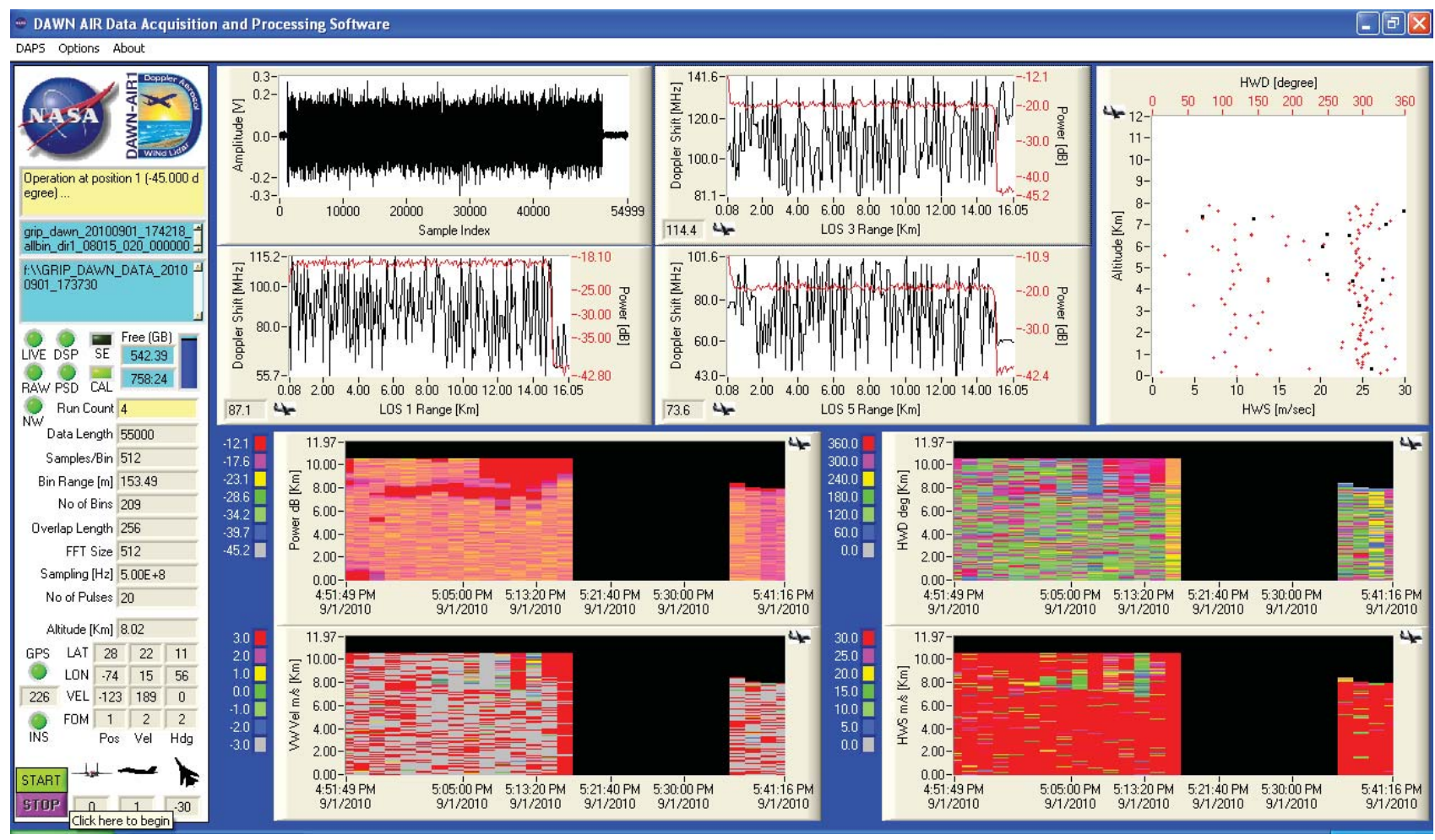

Fig. 2. A screenshot of DAPS in live action

Figure 2 shows a screenshot of DAPS during a real-time operation. The left column panel shows selected important user input parameters as well as real-time status of subsystems. The top five graphs to the right show the raw lidar data, power distribution and Doppler shift estimates of the first, third, and the fifth LOSs, and the horizontal wind speed 
(HWS) and wind direction (HWD) at different altitudes. The bottom four color displays are panoramic displays of power distribution, HWS, HWD, and vertical wind velocity for an extensive period of observation.

\section{SPECTRAL ANALYSIS IN AIRBORNE WIND PROFILING ALGORITHM}

In processing airborne lidar data for wind profiling, spectral analyses are the most accuracy-driven and time critical processes, especially during the real-time processing. The computation of fast Fourier transform (FFT) is the stepping stone for further data processing to estimate wind parameters. Figure 3 shows the concept of lidar data processing, and the spectral analyses involve FFT computation and peak detection. Typically, the length of each lidar pulse sample collection is 50,000 - 60,000, and the monitoring pulse is included in the beginning of each lidar return. The frequency spectrum of the monitoring pulse indicates the quality of each lidar pulse and is used to determine either the lidar return will be used for further processing or discarded. Also, the peak frequency of each monitoring pulse is used to align the spectra of data in all range bins before averaging occurs. Caution must be exercised to maintain high degree of accuracy in those processes.

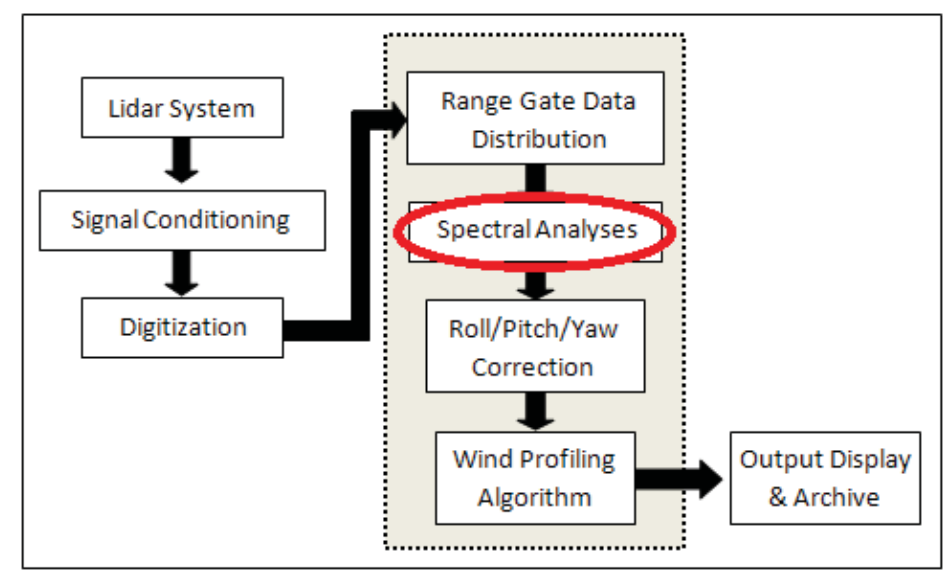

Fig. 3. Wind profiling procedures for DAWN AIR airborne lidar data.

\section{NOISE WHITENING METHODS}

The noise whitening methods that DAPDS implemented are based on background instrument noise floor elimination. The FFT of the data acquired from the lidar system shows recurring peaks when the laser beam scatter from aerosol particles is detected. The instrument noise raises the peaks to be detected unevenly in the frequency bandwidth of interest, and the elimination of this noise is essential to perform the peak detection accurately [13].

Background noise data without lasing the lidar system were acquired for noise spectra estimation. 155 files of background noise were used where each file contained 20 accumulated lidar pulses. They were divided into segments of the size of FFT, and the FFT of each segment was computed and averaged. The periodogram was used to map the noise data into a power spectrum in $\mathrm{dB}$, and is defined by $10 * \log _{10}\left(|\mathrm{FFT}|^{2} / N\right)$, where $N$ is the number of data points in the FFT before zero padding. The resulting background noise spectra are shown in Figure 4 for two different FFT sizes: 512 and 4,096. The comparison of the two different FFT sizes shows that the averaged periodogram is independent of the FFT size, representing the noise floor regardless of the FFT size. Note that the noise is not white, which makes typical statistical and non-statistical noise whitening methods for white noise are not applicable to this case.

The dropsonde data from the GRIP campaign are used as the quality assurance measure of the DAWN AIR wind profiling technique. A dropsonde is a weather probing device designed to be dropped from a airplane, and it transmits weather measurements as it falls until it reaches the ground. Dropsonde data are provided in a text file that contains header information such as date, time, location, etc. followed by columns of data including speed, direction, and altitude. 
LabVIEW was used to compare the DAWN AIR wind parameters with dropsonde data. In order to do so, the dropsonde data had to be imported from a text file into a numerical array in LabVIEW. First, the header was removed, leaving ASCII numeric characters, which were imported into array rows containing speed and direction at a specific altitude. The values of speed, direction, and altitude in all rows were collected into three numeric arrays. Bad data, which were marked by -999, were filtered out.

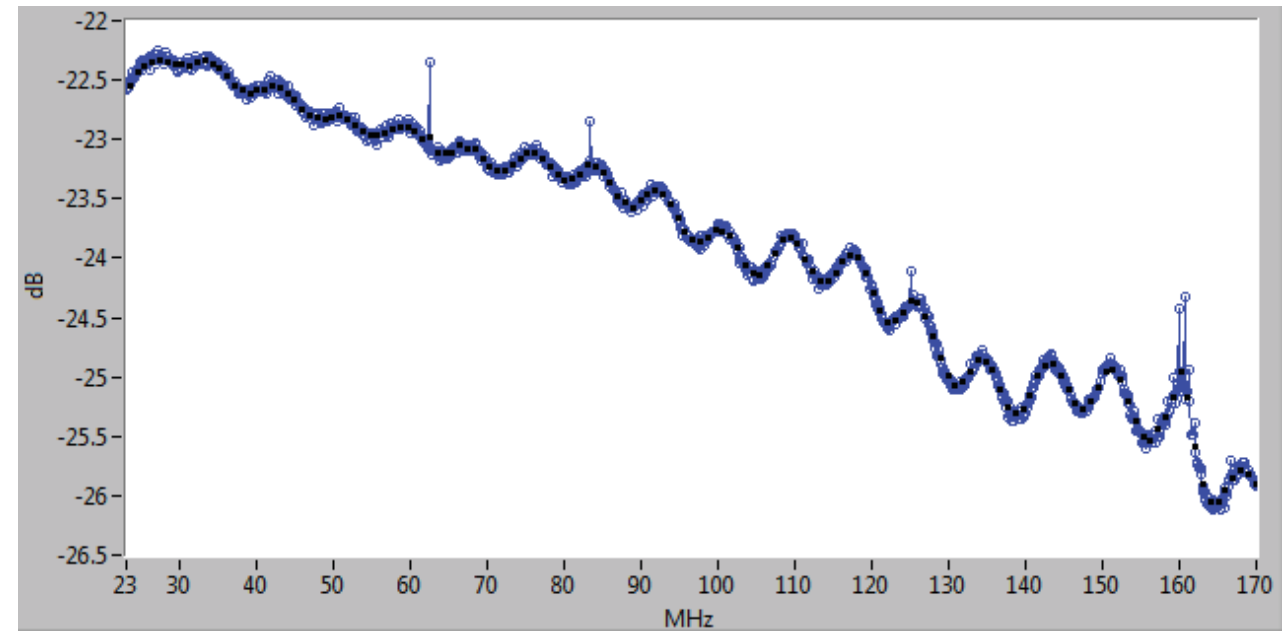

Fig. 4. Noise floor spectra for FFT sizes of 512 (solid line) and 4096 (line with circles). There are no distinguishable differences between the two, indicating the estimated noise floor represents well the spectral behavior of the background noise regardless of the size of FFT.

The LabVIEW program compares the dropsonde data with the DAWN AIR wind parameters and the screenshots of sample comparisons are shown in Figures 5 and 6. The data used to generate the figures were acquired at 17:20:11 Zulu on September 1, 2010. 55,000 samples of lidar data were acquired at each LOS. At each LOS, 20 pulses were accumulated at $10 \mathrm{~Hz}$ trigger rate.
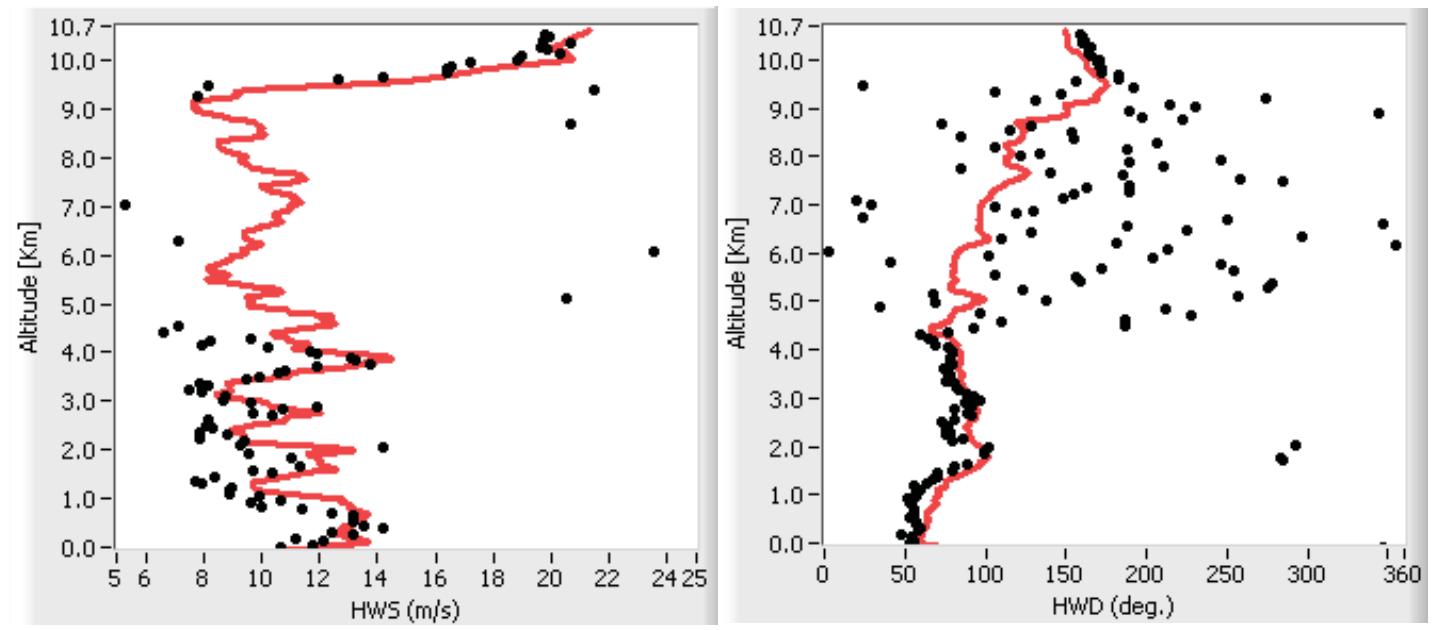

Fig. 5. Examples of dropsonde and DAWN AIR wind product comparisons. Horizontal Wind Speed (HWS) and Horizontal Wind Direction $($ HWD). FFT size $=2048$. Averaged noise floor for noise whitening. Line $=$ Dropsonde, Dots $=$ DAWN AIR . 

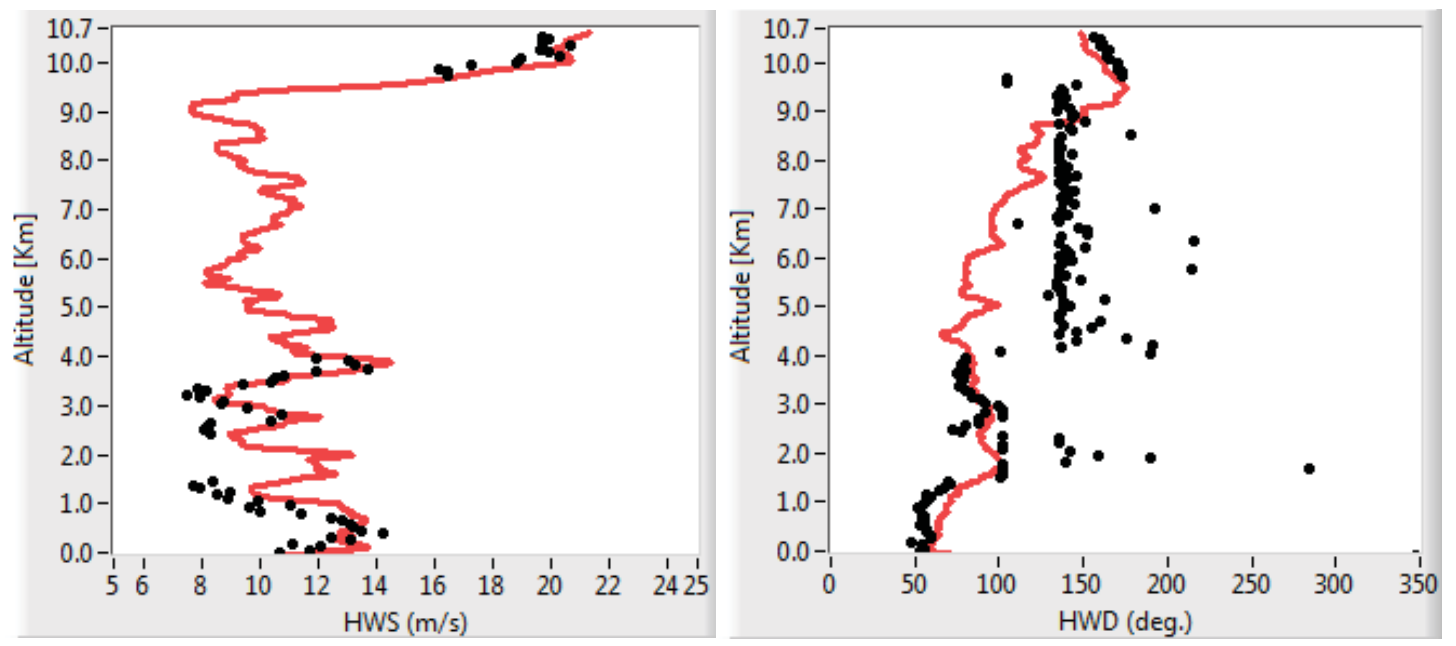

Fig. 6. Examples of dropsonde and DAWN AIR wind product comparisons. Horizontal Wind Speed (HWS) and Horizontal Wind Direction $($ HWD). FFT size $=2048$. Exact noise floor for noise whitening. Line $=$ Dropsonde, Dots = DAWN AIR.

Dropsonde recorded data every half a second during its 8 minute downfall time to ground while DAWN AIR wind profiling is almost instantaneous, which creates a serious altitude resolution discrepancies. In case of 55,000 data points per pulse and a FFT of 512 with a fifty percent overlap, there were 209 range bins or 209 wind parameter values from DAWN AIR. The LabVIEW program takes the DAWN AIR altitude values for each range bin and matches them with the closest altitude recorded by dropsonde by interpolating the two closest altitude values.

Figures 5 and 6 show the comparisons of dropsonde data with DAWN AIR wind parameter estimates for two different noise whitening methods: one with the averaged noise spectral floor and the other with the exact noise spectral floor. As for the averaged noise floor method, a first-order polynomial fitting is performed to represent the noise floor, and is used

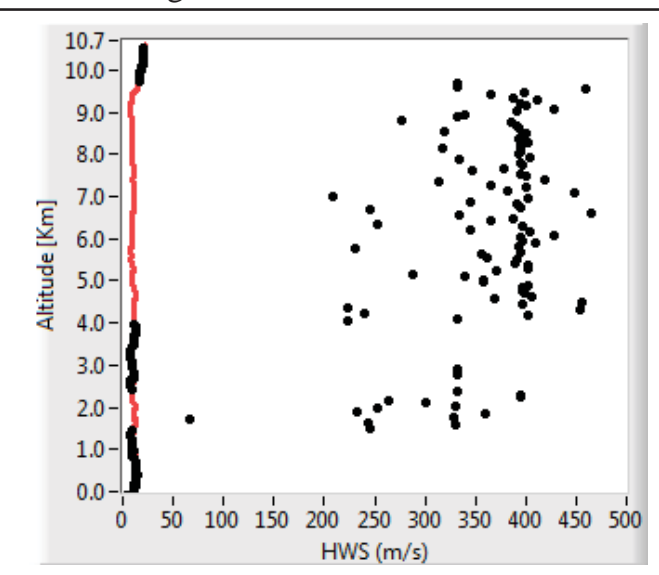

Fig. 7. HWS in Fig. 6 in different $x$ scale for noise whitening. In the exact noise floor method, the noise floor shown in Figure 4 is used for noise whitening. The two wind parameters are compared in this case: HWS and HWD. The size of FFT was 2,048 with zero padding. The comparisons show that normalizing the lidar return spectra with the averaged noise spectra manages to provide wind parameter estimates when the signal-tonoise-ratio (SNR) is low. (4 - $9.5 \mathrm{~km}$ altitude approximately) Where the SNR is relatively strong, both noise whitening methods seem to return the same wind parameter estimates. The weak SNR can be due to clouds or short of atmospheric aerosol layers.

Figure 7 shows the HWS in Figure 6 in different $x$ scale. Where the SNR is low near $4-9.5 \mathrm{~km}$ altitude, the noise whitening using the exact noise spectral floor returns erroneous HWS estimates. Due to the low SNR, normalizing the noise by spiky exact noise spectral floor creates spurious peaks, which will fail the peak detection process. As a result, ill-fated HWS estimates return congregated wrong HWDs in Figure 6.

\section{CONCLUSION}

Spectral analysis is one of the accuracy-critical steps in airborne wind profiling algorithm for DAWN AIR, and noise whitening is an imperative procedure for correct peak detection. Two different methods of noise whitening are 
reviewed. First, the noise spectra were estimated using instrument noise data only. Second, the spectra of DAWN AIR wind lidar data were normalized by the estimate noise spectra. One method is to apply the first-order polynomial fitting to the noise floor to represent the instrument background noise. The other is to use the noise spectra without the fitting. The two wind parameters HWS and HWD were compared with dropsonde data using both methods. We find that the method with the first-order polynomial fitting manages to return decent wind products in low SNR environment.

\section{ACKNOWLEDGMENT}

The authors are grateful for support from NASA Science Mission Directorate (SMD), the SMD Airborne Instrument Technology Transition program, the NASA SMD Earth Science Technology Office (ESTO) Instrument Incubator Program, the ESTO Laser Risk Reduction Program, Langley Research Center (LaRC), and the LaRC Engineering Directorate.

\section{BIBLIOGRAPHY}

[1] J. Y. Beyon, G. J. Koch, and M. J. Kavaya "Development of the Data Acquisition and Processing System for a Pulsed 2-Micron Coherent Doppler Lidar System,” in Proc. of the SPIE Asia-Pacific Remote Sensing (7860-9), Incheon, Republic of Korea, October, 2010.

[2] J. Y. Beyon, G. J. Koch, and M. J. Kavaya "Data Acquisition and Processing System for Airborne Wind Profiling with a Pulsed, 2-Micron, Coherent-Detection, Doppler Lidar System,” in Proc. of the Earth Science Technology Forum 2010, Arlington, VA, June, 2010.

[3] G.J. Koch, J.Y. Beyon, P.J. Petzar, M. Petros, J. Yu, B.C. Trieu, M.J. Kavaya, U.N. Singh, E.A. Modlin, B.W. Barnes, and B.B. Demoz, "Field Testing of a High-Energy 2-um Doppler Lidar," Journal of Applied Remote Sensing 4, 043512, 2010.

[4] K. Vermeesch, G. Koch, B. Gentry, T. Bacha, H. Chen, and B. Demoz, "Comparisons of Ground-Based, Radiosonde, and Aircraft Wind Measurements at the Howard University Beltsville Research Site," Wind Lidar Working Group Meeting, June, 2009.

[5] J. Y. Beyon, G. J. Koch, M. J. Kavaya, and M. Sahota, "Comparison of theoretical and empirical statistics of wind measurements with validation lidar (VALIDAR)," in Proc. of the Defense and Security Symposium 2008(6968-59), Orlando, FL, March, 2008.

[6] J. Y. Beyon, G. J. Koch, M. J. Kavaya, and M. Sahota, "Comparison of theoretical and empirical statistics of wind measurements with validation lidar (VALIDAR)," in Proc. of the Defense and Security Symposium 2008(6968-59), Orlando, FL, March, 2008.

[7] J. Y. Beyon and G. J. Koch, "Novel nonlinear adaptive Doppler-shift estimation technique for the coherent Doppler validation lidar,” Optical Engineering, Vol. 46, No. 1, pp. 016002-1 - 016002-10, January 2007.

[8] J. Y. Beyon, G. J. Koch, and S. Ismail "Signal processing techniques for heterodyne differential absorption lidar,” in Proc. of the Defense and Security Symposium 2007 (6567-53), Orlando, FL, April, 2007.

[9] G.J. Koch, J.Y. Beyon, B.W. Barnes, M. Petros, J. Yu, F. Amzajerdian, M.J. Kavaya, and U.N. Singh, "HighEnergy 2- $\mu m$ Doppler Lidar for Wind Measurements," Optical Engineering, Vol. 46(11), pp. 116201-1 116201-14, November, 2007.

[10] J. Y. Beyon and G. J. Koch, "Novel Nonlinear Adaptive Doppler Shift Estimation Technique (NADSET) for the Coherent Doppler Lidar System VALIDAR," in Proc. of the Defense and Security Symposium 2006 (62361), Orlando, FL, April, 2006.

[11] J. Y. Beyon and G. J. Koch, "Resolution Study of Wind Parameter Estimates by a Coherent Doppler Lidar System,” in Proc. of the Defense and Security Symposium 2006 (6214-3), Orlando, FL, April, 2006.

[12] J. Y. Beyon and G. J. Koch, "Wind Profiling by a Coherent Doppler Lidar System VALIDAR with a Subspace 
Decomposition Approach,” in Proc. of the Defense and Security Symposium 2006 (6236-5), Orlando, FL, April, 2006.

[13] J. Y. Beyon, G. J. Koch, and Z. Li "Noise Normalization and Windowing Functions for VALIDAR in Wind Parameter Estimation," in Proc. of the Defense and Security Symposium 2006 (6214-4), Orlando, FL, April, 2006.

[14] J. Yu, B.C. Trieu, E.A. Modlin, U.N. Singh, M.J. Kavaya, S. Chen, Y. Bai, P.J. Petzar, “1 J/pulse Q-switched 2 um solid-state laser,” Optics Letters 31, 462-462, 2006. 